\title{
Structure and properties of the vacuum of the Twisted Eguchi-Kawai model
}

\author{
Hélvio Vairinhos* \\ University of Oxford \\ E-mail: helvio@thphys.ox.ac.uk \\ Michael Teper \\ University of Oxford \\ E-mail: teper@thphys.ox.ac.uk
}

\begin{abstract}
We investigate numerically the phase structure of the Twisted Eguchi-Kawai (TEK) model in four dimensions. In the numerical simulations of the zero-temperature TEK model (using a symmetric twist) we observe the existence of new phases that break its $Z_{N}^{4}$ symmetry at intermediate lattice couplings and for large $\mathrm{SU}(N)$ gauge groups. This effect can be explained by the contribution of diagonal configurations with collapsed eigenvalues, which are particular cases of "generalised fluxons". We also investigate finite-temperature versions of the TEK model using anisotropic lattice couplings, where in particular we find van Baal fluxons contributing at large anisotropies.
\end{abstract}

The XXV International Symposium on Lattice Field Theory

July 30-4 August 2007

Regensburg, Germany

\footnotetext{
${ }^{*}$ Speaker.
} 


\section{Introduction}

Eguchi-Kawai (EK) reduced models offer a potentially useful alternative to the usual approach to large- $N$ extrapolations in lattice gauge theory. In four dimensions, they are defined as $\mathrm{SU}(N)$ Wilson lattice gauge theories living on a $1^{4}$ lattice. In the large- $N$ limit, these models were conjectured [1] to be equivalent to $\mathrm{SU}(N)$ Wilson lattice gauge theories living on an infinite lattice; this equivalence is known as EK correspondence. In practice, the absence of space-time degrees of freedom in EK models allows the simulation of relatively large gauge groups with moderate computational effort, which alludes to a faster convergence to the planar limit. However, the EK correspondence doesn't always hold: in the original EK model [1], i.e. with periodic boundary conditions, the EK correspondence is known to break at weak coupling. In the EK model with twisted boundary conditions [2], on the other hand, it is generally assumed that the problems that affect the untwisted EK model are absent, and consequently that the EK correspondence holds for all couplings. In this talk we summarise the results of our study [3] of the properties of some four-dimensional TEK models, which have strong implications for the validity of the EK correspondence.

\section{The EK model and its vacuum}

The action of the four-dimensional EK model [1] is given by:

$$
S_{\mathrm{EK}}(U)=b N \sum_{\mu \neq v}^{4} \operatorname{Tr}\left\{\mathbb{1}-U_{\mu} U_{v} U_{\mu}^{\dagger} U_{v}^{\dagger}\right\}
$$

where $b=\frac{1}{g^{2} N}$ is the inverse 't Hooft coupling, and $U_{\mu} \in \mathrm{SU}(N)$ are the link variables. The EK action has a gauge symmetry:

$$
U_{\mu} \mapsto V U_{\mu} V^{\dagger}, V \in \mathrm{SU}(N)
$$

and also a $Z_{N}^{4}$ symmetry:

$$
U_{\mu} \mapsto z_{\mu} U_{\mu}, z_{\mu} \in Z_{N}
$$

The EK correspondence [1] states that $\mathrm{SU}(N)$ Wilson lattice gauge theories living on an infinite lattice should be equivalent to $\mathrm{SU}(N)$ EK models in the large- $N$ limit, in the sense that expectation values of corresponding operators coincide in that limit, i.e. $\left\langle\mathscr{O}\left[U_{\mu}(x)\right]\right\rangle_{\mathrm{W}} \stackrel{N \rightarrow \infty}{=}\left\langle\mathscr{O}\left[U_{\mu}\right]\right\rangle_{\mathrm{EK}}$. This is a consequence of the fact that the large- $N$ Schwinger-Dyson (SD) equations of the original Wilson theory and its EK reduced model coincide. More precisely, the SD equations have the same form in both theories, except for contact terms that are specific of the EK model. These contact terms consist of expectation values of open lines; they vanish exactly in the original Wilson theory (due to gauge symmetry), but not necessarily in the EK model (since the gauge symmetry (2.2) is a similarity transformation of the link variables). However, in the EK model the open lines are not invariant under the $Z_{N}^{4}$ symmetry (2.3). So, unless the centre symmetry is spontaneously broken, the expectation values of open lines is zero and the EK correspondence holds nonperturbatively.

In the strong coupling regime, $b \rightarrow 0$, the Haar measure dominates the partition function of the EK model. This measure has a repulsive effect on the eigenvalues of the link variables, which results in an essentially uniform distribution of eigenvalues over the unit circle ${ }^{1}$ consequently,

\footnotetext{
${ }^{1}$ Recall that eigenvalues of $\mathrm{SU}(N)$ matrices are pure $\mathrm{U}(1)$ phases.
} 
the traces of link variables (and other open lines) have zero expectation value, and hence the EK correspondence holds in the weak coupling regime.

In the weak coupling regime, $b \rightarrow \infty$, small fluctuations around the absolute minimum of the EK action (2.1) dominates the partition function. Let $U_{\mu}=\Omega_{\mu} e^{-X_{\mu}}$ denote a perturbation (labelled by anti-Hermitian matrices $X_{\mu}$ ) of the reduced link variables around an extremum $\Omega_{\mu}$ of the EK action. The stationarity points of the action $\left(\delta S_{\mathrm{EK}}=0\right)$ are $\mathrm{SU}(N)$ diagonal matrices, $\Omega_{\mu}=\operatorname{diag}\left\{e^{i \varphi_{1}}, \ldots, e^{i \varphi_{N}}\right\}$. The trace of a reduced link variable averaged over the classical vacuum manifold is obviously zero. However, the second variation of the EK action around these diagonal extrema is:

$$
\delta^{2} S_{\mathrm{EK}} \propto-\sum_{\mu \neq v}^{4} \operatorname{Tr}\left\{\left(\Omega_{\mu} X_{\nu} \Omega_{\mu}^{\dagger}-X_{v}\right)-\left(\Omega_{v} X_{\mu} \Omega_{v}^{\dagger}-X_{\mu}\right)\right\}^{2} \geq 0
$$

When the extremum is a centre element, $\Omega_{\mu}=z_{\mu} \mathbb{1} \in Z_{N}$, we have $\delta^{2} S_{\mathrm{EK}}\left(z_{\mu} \mathbb{1}\right) \equiv 0$, while for general diagonal matrices we have $\delta^{2} S_{\mathrm{EK}}\left(\Omega_{\mu}\right)>0$. This means that the elements of the centre, having lower action, are the true vacua of the quantum EK model. These vacua break the $Z_{N}^{4}$ symmetry spontaneously, and hence the EK correspondence does not hold in the continuum limit.

\section{The TEK model and its vacuum}

An elegant way to avoid the spontaneous breaking of the $Z_{N}^{4}$ symmetry in the EK model is to introduce twisted boundary conditions in the reduced $1^{4}$ lattice, as pioneered by González-Arroyo and Okawa [2]. The action of the twisted EK (TEK) model is given by:

$$
S_{\mathrm{TEK}}(U)=b N \sum_{\mu \neq v}^{4} \operatorname{Tr}\left(\mathbb{1}-z_{\mu \nu} U_{\mu} U_{v} U_{\mu}^{\dagger} U_{v}^{\dagger}\right)
$$

where $z_{\mu v}=e^{i \frac{2 \pi}{N} n_{\mu v}}$, and $n_{\mu v}=-n_{v \mu}=L$, for all $\mu<v$, is the symmetric twist tensor of GonzálezArroyo and Okawa [2], with $N=L^{2}$. The EK correspondence states that the $\mathrm{SU}(N)$ TEK model with symmetric twist tensor has the same planar limit as the $\mathrm{SU}(N)$ Wilson lattice gauge theory living in a periodic $L^{4}$ lattice.

In the strong coupling regime, the Haar measure dominates the TEK partition function. This implies, like in the EK model, that the expectation values of the traces of open lines are zero.

In the weak coupling regime, small fluctuations around the classical minimum of (3.1) dominate the TEK partition function. This classical minimum, known as the twist-eater, is the configuration $U_{\mu}=\Gamma_{\mu}$ that solves the equation $e^{i \frac{2 \pi}{L}} \Gamma_{\mu} \Gamma_{v} \Gamma_{\mu}^{\dagger} \Gamma_{v}^{\dagger}=1$; it is given, up to gauge rotations (2.2) and $Z_{N}$ shifts (2.3), by $\Gamma_{\mu} \sim \bigoplus^{L} \operatorname{diag}\left\{1, e^{i \frac{2 \pi}{L}}, \ldots, e^{i \frac{2 \pi}{L}(L-1)}\right\}$. Small fluctuations around $\Gamma_{\mu}$ correspond to small fluctuations around these classical eigenvalues. The distribution of eigenvalues of a link variable is then $Z_{L}$-symmetric over the unit circle, and hence its trace is automatically zero. In sum, small fluctuations around the classical minimum of the TEK action preserve enough of the $Z_{N}^{4}$ symmetry (namely $Z_{L}^{4}$ ), for the EK correspondence to hold in the weak coupling regime of the TEK model (unlike in the untwisted EK model).

It is generally assumed that the $Z_{N}^{4}$ symmetry of the TEK model is intact for all couplings. However, since the arguments used for the weak and strong coupling regimes are distinct, there is no a priori reason why the centre symmetry should not be broken at intermediate couplings. 

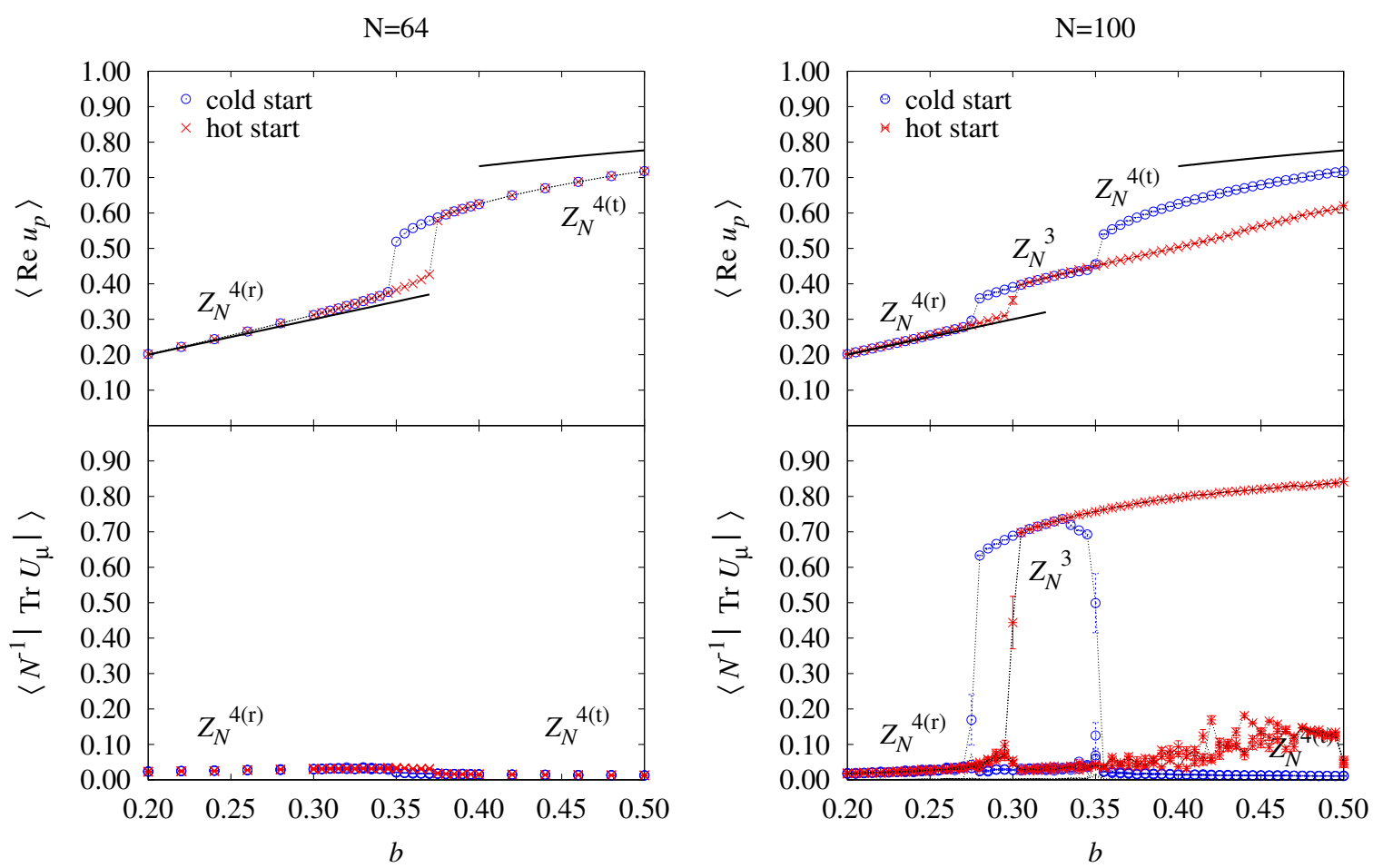

Figure 1: Expectation values of the plaquette, $u_{p}$, (top) and traced link variables, $\operatorname{Tr} U_{\mu}$, (bottom) in the $\mathrm{SU}(N)$ TEK models for $N=64$ (left) and $N=100$ (right). $Z_{N}^{k}$ denotes a phase with $k$ preserved $Z_{N}$ symmetries; $Z_{N}^{4(r)}$ and $Z_{N}^{4(t)}$ denote phases that are $Z_{N}^{4}$-symmetric due to the randomisation from the Haar measure $(r)$ or due to fluctuations around the twist-eater vacuum $(t)$, respectively.

\section{4. $Z_{N}^{4}$ symmetry breaking}

We performed numerical simulations of the SU $(N)$ TEK model with symmetric twist for a wide range of gauge groups, namely $25 \leq N \leq 256$. For $N \leq 81$, the TEK model has properties similar to the ones of the original Wilson theory: it has a strong first-order bulk transition at $b \approx 0.36$, and agrees with the strong- and weak-coupling expansions of the plaquette (left-top graph in Fig.1). In sum, the EK correspondence seems to hold without restrictions, which is also suggested by the fact that the trace of reduced link variables is zero for all couplings (left-bottom graph in Fig.1). However, for $N \geq 100$, the $Z_{N}^{4}$ symmetry of the TEK model is spontaneously broken at intermediate couplings; this is manifest by at least one of the link variables acquiring a non-zero trace (rightbottom graph in Fig.1). In hot start simulations, we also observe that once the reduced lattice falls into a $Z_{N}$-breaking vacuum, it remains there for the whole simulation, even for very large values of the coupling $(b \approx 20)$. This metastability suggests the existence of stable extrema of the TEK action that do not preserve its $Z_{N}^{4}$ symmetry.

We also observe that the four independent $Z_{N}$ symmetries of the TEK model break independently in a cascading way: $Z_{N}^{4} \rightarrow Z_{N}^{3} \rightarrow Z_{N}^{2} \rightarrow Z_{N}^{1} \rightarrow Z_{N}^{0}$ (Fig.2). These transitions are similar to the ones observed on a continuum torus by Narayanan and Neuberger [5]. However, they are not the physical transitions of the original Wilson theory, but instead the transitions associated with the $1^{4}$ lattice (where the link variables play the role of Polyakov loops). Across each one of these transition, the eigenvalues of one of the link variables collapse onto an element of $Z_{N}$, which con- 

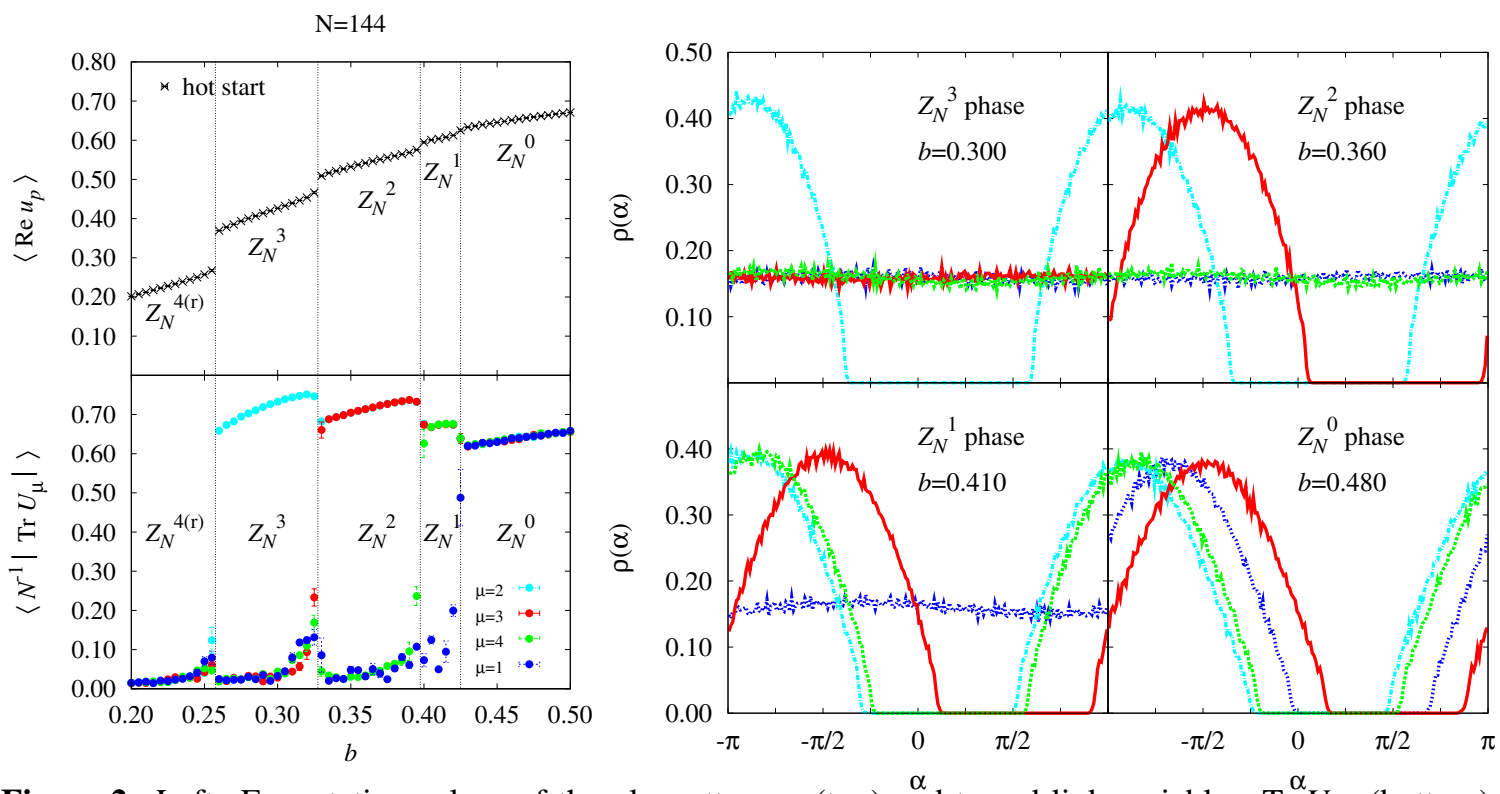

Figure 2: Left: Expectation values of the plaquette, $u_{p}$, (top) and traced link variables, $\operatorname{Tr}^{\alpha} U_{\mu}$, (bottom) in the $\mathrm{SU}(N)$ TEK models for $N=144$. Right: Eigenvalue distributions, $\rho(\alpha)$, of the link variables in the different $Z_{N}^{k}$-symmetric phases of the SU(144) TEK model $(k=0,1,2,3)$.

sequently leads to a non-zero expectation value for its trace. This suggests that the stable relative extrema of the TEK action that break the $Z_{N}^{4}$ symmetry are centre configurations.

The existence of $Z_{N}$-breaking phases in the TEK model has been confirmed independently by Ishikawa and Okawa [6], and has also been also observed in the context of discrete noncommutative gauge theories [7].

\section{Interpretation}

A class of stable extrema of the TEK action (3.1) are the unitary solutions $\Omega_{\mu}$ of the equation $e^{i \frac{2 \pi}{L}} \Omega_{\mu} \Omega_{v} \Omega_{\mu}^{\dagger} \Omega_{v}^{\dagger}=e^{i \frac{2 \pi}{N} k_{\mu v}} \mathbb{1}$, where $\cos \left(\frac{2 \pi}{N} k_{\mu v}\right) \geq 0$ for all $\mu<v$ (stability condition). These solutions are called 'fluxons' 2 [4]. Solutions of the same 'fluxon level', $k=\frac{1}{2} \sum_{\mu<\nu}^{4} k_{\mu \nu}^{2} \in \mathbb{Z}$, are gauge-equivalent and have the same classical action, $S_{\mathrm{TEK}} \propto k$. The classical minimum of the TEK action - the twist-eater - is the solution for the special case $k_{\mu \nu} \equiv 0$.

If we consider the case $k_{\mu v}=L$, then the stationarity equation is $\Omega_{\mu} \Omega_{v} \Omega_{\mu}^{\dagger} \Omega_{v}^{\dagger}=\mathbb{1}$, whose solution manifold is the set of all diagonal matrices. This is a stable minimum of the TEK action. As in the case of the untwisted EK model (Section 2), fluctuations around this extremum result in the centre configurations $\left(z_{\mu} \mathbb{1}\right)$ having a lower action than the general diagonal matrix. Therefore, the $Z_{N}^{4}$ symmetry is also spontaneously broken in these extrema. The numerical data suggests that at intermediate couplings the TEK model is dominated by diagonal matrices, even though these extrema do not survive the large- $N$ limit (because $S_{\mathrm{TEK}} \propto N$ ). However, at intermediate couplings and finite $N$, the magnitude of the fluctuations $X_{\mu}$ around the twist-eater may be large enough to overcome the barriers between extrema and eventually tunnel to a centre configuration. While the

\footnotetext{
${ }^{2}$ Normally, the term 'fluxon' is only used when the solution for a given $k_{\mu v}$ gives a non-zero contribution to the partition function in the $N \rightarrow \infty$ limit. Here we abuse the language and call 'fluxon' any solution of this equation.
} 


\begin{tabular}{|c|c|c|c|c|c|c|c|c|c|}
\hline$N$ & & & & & $\leftarrow$ & & & & \\
\hline 100 & {$\left[Z_{N}^{4(r)}\right]$} & $0.275(5)$ & {$\left[Z_{N}^{3}\right]$} & & & $0.350(5)$ & & & {$\left[Z_{N}^{4(t)}\right]$} \\
\hline 121 & {$\left[Z_{N}^{4(r)}\right]$} & $0.250(5)$ & {$\left[Z_{N}^{3}\right]$} & & $0.325(5)$ & & {$\left[Z_{N}^{0}\right]$} & $0.360(5)$ & {$\left[Z_{N}^{4(t)}\right]$} \\
\hline 144 & {$\left[Z_{N}^{4(r)}\right]$} & $0.235(5)$ & {$\left[Z_{N}^{3}\right]$} & $0.275(5)$ & {$\left[Z_{N}^{2}\right]$} & $0.325(5)$ & {$\left[Z_{N}^{0}\right]$} & $0.370(5)$ & {$\left[Z_{N}^{4(t)}\right]$} \\
\hline 169 & {$\left[Z_{N}^{4(r)}\right]$} & $0.235(15)$ & {$\left[Z_{N}^{3}\right]$} & $0.25(3)$ & {$\left[Z_{N}^{2}\right]$} & $0.355(15)$ & {$\left[Z_{N}^{0}\right]$} & $0.37(3)$ & {$\left[Z_{N}^{4(t)}\right]$} \\
\hline 196 & {$\left[Z_{N}^{4(r)}\right]$} & & $0.235(15)$ & & {$\left[Z_{N}^{2}\right]$} & $0.28(3)$ & {$\left[Z_{N}^{0}\right]$} & $0.385(15)$ & {$\left[Z_{N}^{4(t)}\right]$} \\
\hline 225 & {$\left[Z_{N}^{4(r)}\right]$} & & $0.22(3)$ & & {$\left[Z_{N}^{2}\right]$} & $0.28(3)$ & {$\left[Z_{N}^{0}\right]$} & $0.415(15)$ & {$\left[Z_{N}^{4(t)}\right]$} \\
\hline 256 & {$\left[Z_{N}^{4(r)}\right]$} & $0.205(15)$ & {$\left[Z_{N}^{3}\right]$} & $0.235(15)$ & {$\left[Z_{N}^{2}\right]$} & $0.28(3)$ & {$\left[Z_{N}^{0}\right]$} & $0.43(3)$ & {$\left[Z_{N}^{4(t)}\right]$} \\
\hline
\end{tabular}

Table 1: Critical values of $b$ associated with the breaking/restoration of one (or more) $Z_{N}$ symmetries of the TEK model, for cold start simulations. $\left[Z_{N}^{k}\right]$ refers to the phase with $k$ unbroken directions; the numerical values denote the critical couplings; a numerical value spanning multiple columns is the critical coupling associated with the simultaneous breaking of more than one $Z_{N}$ symmetry.

contribution to the TEK action from fluctuations around the twist-eater configuration is quadratic, it is of higher order around centre configurations:

$$
\left\{\begin{array}{l}
S_{\mathrm{TEK}}\left(\Gamma_{\mu}\right) \approx b N \sum_{\mu<v}^{4} \operatorname{Tr}\left(F_{\mu v}^{2}\right)+\cdots \equiv O\left(X^{2}\right)+O\left(X^{3}\right) \\
S_{\mathrm{TEK}}\left(z_{\mu} \mathbb{1}\right) \approx 24 b N^{2} \sin ^{2}\left(\frac{\pi}{\sqrt{N}}\right)+\cdots \equiv O\left(X^{0}\right)+O\left(X^{3}\right)
\end{array}\right.
$$

We may imagine that, at some critical coupling, the action for fluctuations around the twist-eater may become larger than the action for fluctuations around a centre configuration, inducing the tunnelling between the two extrema and hence breaking the $Z_{N}^{4}$ symmetry spontaneously.

We also observe that the critical couplings of the $Z_{N}$-breaking transitions (Table 1) show a dependence with $N$ that points to a widening of the $Z_{N}$-broken region for increasing $N$. In addition, we have not been able to detect any evidence for the expected confining/deconfining transition (or any other transitions [5]) at weak couplings.

\section{Anisotropic TEK model and fluxon vacua}

We also simulated anisotropic TEK models, mainly to look for evidence of the large- $N$ confining/deconfining transition at finite temperature. The action of the most general anisotropic TEK model is given by:

$$
S_{\mathrm{TEK}}\left(\xi_{i}, U\right)=b N \sum_{\mu \neq v}^{4} \xi_{\mu v} \operatorname{Tr}\left(\mathbb{1}-z_{\mu v} U_{\mu} U_{v} U_{\mu}^{\dagger} U_{v}^{\dagger}\right)
$$

where $\xi_{\mu v}=\frac{\xi_{\mu} \xi_{v}}{\xi_{\kappa} \xi_{\lambda}}(\kappa \neq \lambda$, and $\kappa, \lambda \neq \mu, v)$, and $\xi_{\mu}=\frac{a_{4}}{a_{\mu}}\left(a_{\mu}\right.$ is the lattice spacing in the $\mu$ direction). For $N \geq 81$, we observe that the $Z_{N}^{4}$ symmetry is broken by centre configurations. For $N \leq 64$, however, different phases appear at intermediate coupling. By analysing the values of the plaquettes and eigenvalue structure of the link variables in these new extrema (Fig.3), we conclude that they are fluxon configurations [4]. We observe intermediate stable configurations belonging to several different fluxon levels, depending on the choice of $\xi_{i}$; in particular, for $\xi_{i}=\xi$, we observe van Baal fluxons [4] for the first time, namely fluxons of the level $k=1$ (Fig.3); for different cases, the fluxons are more general. We again found no evidence for the confining/deconfining transition (or any other transitions [5]) in the anisotropic TEK model. 

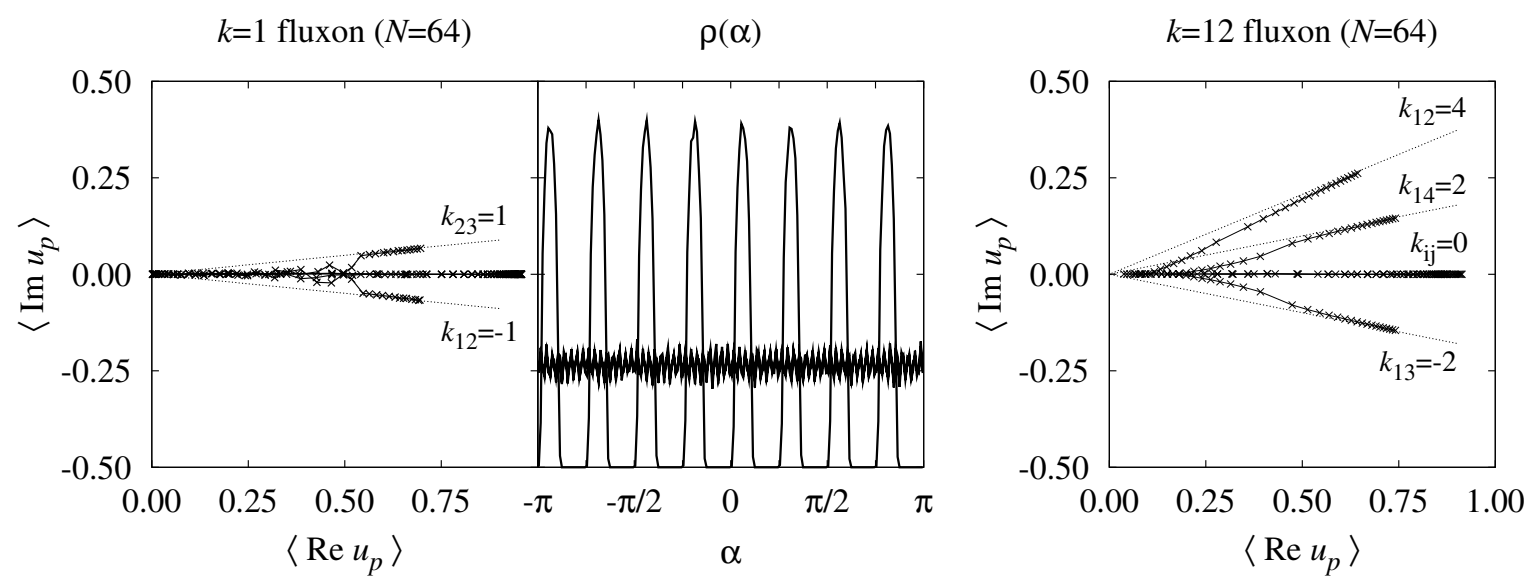

Figure 3: Traces of plaquettes, $u_{p}$, and eigenvalue densities of link variables, $\rho(\alpha)$, in the SU(64) anisotropic TEK model, for different choices of $\xi_{i}: \xi_{i}=0.15$ (left) and $\xi_{i}=(0.50,0.75,1.00)$ (right).

\section{Conclusions}

We showed that for sufficiently large $N$, the $Z_{N}^{4}$ symmetry of $\mathrm{SU}(N)$ TEK models is spontaneously broken at intermediate couplings, where centre configurations dominate. These $Z_{N^{-}}$ breaking phases appear to be extending further into weak coupling as $N$ increases. Even though we cannot yet establish a clear trend for this $N$-dependence, it suggests that the range of couplings of the $Z_{N}$-broken phases may continue to grow and eventually dominate the whole phase diagram of the TEK model in the $N \rightarrow \infty$ limit, thus invalidating the EK correspondence for this model, or at least making it impractical for the study of the physics of large- $N$ gauge theories.

We also observed several fluxon vacua dominating at intermediate couplings in anisotropic TEK models. This shows that the phase diagram of the TEK model is much richer than previously thought. However, no evidence of any transition to a physical phase was observed, which could mean that they are absent or inaccessible.

\section{Acknowledgements}

Our lattice simulations were carried out on PPARC and EPSRC funded computers in Oxford Theoretical Physics. HV is supported by FCT (Portugal) under the grant SFRH/BD/12923/2003.

\section{References}

[1] T. Eguchi and H. Kawai, Phys. Rev. Lett. 48 (1982) 1063.

[2] A. González-Arroyo and M. Okawa, Phys. Rev. D 27 (1983) 2397;

[3] M. Teper and H. Vairinhos, Phys. Lett. B 652 (2007) 359 [hep-th/0612097].

[4] P. van Baal, Commun. Math. Phys. 92 (1983) 1.

[5] R. Narayanan and H. Neuberger, PoS LAT2005 (2006) 005 [hep-lat/0509014].

[6] T. Ishikawa, T. Azeyanagi, M. Hanada and T. Hirata, in these proceedings; M. Okawa, private communication.

[7] W. Bietenholz, A. Bigarini, J. Nishimura, Y. Susaki, A. Torrielli and J. Volkholz, in these proceedings. 